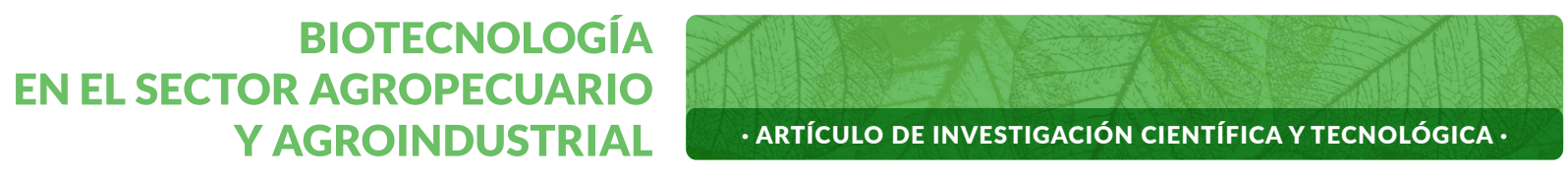

Vol. 16 No 2 · Julio - Diciembre 2018 • ISSN - 1692-3561 · ISSN-e 1909-9959 · doi: http://dx.doi.org/10.18684/bsaa.v16n2.1170

\title{
Propiedades funcionales de almidones de ñame (Dioscorea alata)
}

\section{Funtional properties of starch yam (Dioscorea alata)}

\section{Propriedades funcionais do amido de inhame (Dioscorea alata)}

\author{
JAIRO SALCEDO-MENDOZA', CARLOS GARCÍA-MOGOLLÓN², \\ DAVID SALCEDO-HERNÁNDEZ ${ }^{3}$
}

\section{RESUMEN}

El ñame es un producto característico de la costa Caribe colombiana. El desconocimiento de las propiedades fisicoquímicas del ñame, ha restringido el uso del producto a consumo en fresco y preparaciones tradicionales. La industria de alimentos se encuentra en la búsqueda de almidones nativos que presenten ciertas propiedades específicas de resistencia a altas temperaturas deterioren la estructura del gel de almidón y posibiliten la producción de almidones resistentes. Se estudiaron las propiedades fisicoquímicas, y comportamiento reológico de cinco variedades de ñame (D. alata) (9506-027, 9403-001, 9605-047, 9506-021 y 9506-025) obtenidas del banco de germoplasma de la universidad de Córdoba (Colombia). El contenido de amilosa de los almidones estudiados, estuvieron entre 23,2 y 25,6 p/p. Algunas

Recibido para evaluación: 18 de Febrero de 2017.

Aprobado para publicación: 26 de Julio de 2018.

1 Universidad de Sucre, Programa de Ingeniería Agroindustrial, Grupo de Investigación PADES. Ph.D. Sincelejo, Colombia.

2 Universidad de Sucre, Programa de Ingeniería Agroindustrial, Grupo de Investigación PADES. MSc. Sincelejo, Colombia.

3 Universidad de Córdoba, Programa de Ingeniería de Alimentos. Esp. Evaluación Socio Económica de Proyectos. Montería, Colombia.

Correspondencia: jairo.salcedo@unisucre.edu.co. 
variedades presentaron valores de capacidad de retención de agua superiores al 100\%, posibilitando su uso en la industria alimentaria, dirigido a la producción de productos cárnicos. Todas las variedades presentaron valores bajos de conductividad eléctrica, menores de $80 \mu \mathrm{s} / \mathrm{cm}$. Las viscosidades más altas durante el proceso de calentamiento y enfriamiento son registradas por las variedades 9506-027 y 9403-001, por lo que serían útiles como espesantes para productos alimentarios. Las temperaturas de gelatinización presentados por los almidones de D. alata generalmente están por encima de $83^{\circ} \mathrm{C}$ y se comportaron como fluidos no newtonianos

\section{ABSTRACT}

Yam is a characteristic product of the Colombian Caribbean coast. Ignorance of the physicochemical properties of yams has restricted the use of the product to fresh consumption and traditional preparations. The food industry is in search of native starches that have certain specific properties of resistance to high temperatures that deteriorate the structure of the starch gel and facilitate the production of resistant starches. The physicochemical properties and rheological behavior of five varieties of yam (D. alata) (9506-027, 9403001, 9605-047, 9506-021 and 9506-025) obtained from the germplasm bank of the University of Córdoba were studied. (Colombia). The amylose content of the starches studied was between 23,2 and 25,6 w/w. Some varieties presented water retention capacity values above $100 \%$, making it possible to use them in the food industry, aimed at the production of products. All the varieties presented low values of electrical conductivity, lower than $80 \mu \mathrm{s} / \mathrm{cm}$. The highest viscosities during the heating and cooling process are registered by the varieties 9506-027 and 9403-001, so they would be useful as thickeners for food products. The gelatinization temperatures presented by $D$. alata starches are generally above $83^{\circ} \mathrm{C}$ and behaved as non-Newtonian fluids.

\section{RESUMO}

O inhame é um produto característico da costa do Caribe colombiano. A ignorância das propriedades físico-químicas do inhame restringiu o uso do produto ao consumo in natura e às preparações tradicionais. A indústria alimentícia está em busca de amidos nativos que possuem certas propriedades específicas de resistência a altas temperaturas que deterioram a estrutura do gel de amido e facilitam a produção de amidos resistentes. Foram estudadas as propriedades físico-químicas e o comportamento reológico de cinco variedades de inhame (D. alata) (9506-027, 9403-001, 9605-047, 9506-021 e 9506-025) obtidas do banco de germoplasma da Universidade de Córdoba. (Colômbia). O teor de amilose dos amidos estudados foi entre 23,2 e 25,6 $\mathrm{p} / \mathrm{p}$. Algumas variedades apresentaram valores de capacidade de retenção de água acima de 100\%, possibilitando sua utilização na indústria de alimentos, visando a produção de produtos. Todas as variedades apresentaram baixos valores de condutividade elétrica, inferiores a $80 \mu \mathrm{s} / \mathrm{cm}$. As maiores viscosidades durante o processo de aquecimento e resfriamento são registradas pelas variedades 9506-027 e 9403-001, então elas seriam úteis como espessantes para produtos alimentícios. As temperaturas de gelatinização apresentadas pelos

\section{PALABRAS CLAVES:}

D. alata, Pseudoplástico, Reología, Gelatinización, Capacidad de retención de agua.

\section{KEY WORDS:}

D. alata, Pseudoplastic, Rheology, Gelatinization, Water holding capacity.

\section{PALAVRAS-CHAVE:}

D. alata, Pseudoplástico, Reologia, Gelatinização, Capacidade de retenção de água. 
amidos de D. alata são geralmente superiores $a 83^{\circ} \mathrm{C} e$ comportam-se como fluidos não newtonianos.

\section{INTRODUCCIÓN}

El ñame, es un cultivo de pequeños y medianos agricultores que constituye en muchas regiones de Colombia, la principal fuente de empleo rural y oferta de alimento a sus pobladores. En Colombia, se cultivaron alrededor de 314991 toneladas en el año 2013, con un rendimiento de 10,7 toneladas por hectárea sembrada [1]. La estructura y propiedades físico químicas de almidones de tubérculos y raíces han sido estudiadas extensivamente [2]; sin embargo, poca atención se ha prestado a especies de ñame (Dioscorea spp). En la Costa Atlántica existen cinco especies de ñame: el ñame criollo (D. alata), ñame espino (D. rotundata), ñame papa (D. bulbífera), ñame azúcar (D. esculenta) y ñampin (D. trífida), entre las cuales se encuentran más de 100 variedades siendo $D$. alata y $D$. rotundata las de mayor consumo. El ñame es un producto característico de la franja del trópico y forma parte de la dieta diaria de las personas que viven en estas regiones. El desconocimiento de las propiedades fisicoquímicas del ñame, ha restringido el uso del producto a consumo en fresco y preparaciones tradicionales [3].

Actualmente, la industria de alimentos se encuentra en la búsqueda de almidones nativos que presenten ciertas propiedades específicas de tolerancia a diferentes tratamientos industriales estresantes que deterioren la estructura del gel de almidón, como, por ejemplo: resistencia a la acidez, resistencia a la esterilización y esfuerzos mecánicos y térmicos [2, 4]. Las harinas y almidones obtenidos a partir de diferentes variedades de ñame presentan ciertas propiedades funcionales, entre las cuales se destacan la ausencia de un máximo de viscosidad, la estabilidad de las suspensiones a elevadas temperaturas y bajos valores de $\mathrm{pH}$, que podrían ser utilizados en la elaboración de productos que deben mantener su viscosidad estable durante una fase de calentamiento constante, como, por ejemplo, las mezclas para sopas y pudines instantáneos [5, 6]. Los procesos de transformación de ñame son escasos, el $78 \%$ de la producción de ñame en Colombia está destinado al consumo en fresco desconociéndose sus aplicaciones industriales. Lo anterior es causado por la falta de investigaciones orientadas a la valoración del ñame como materia prima agroindustrial [7], teniendo en cuenta la estimación de sus propiedades fisicoquímicas, que permitan resolver interrogantes como: cuales pueden ser las aplicaciones de los almidones de las variedades de la especie Diocorea alata, que puedan generar un valor agregado al producto.

En este estudio se evaluaron las propiedades fisicoquímicas y funcionales como capacidad de retención de agua, solubilidades, conductividad eléctrica, sinéresis, contenido de amilosa y comportamiento reológico de los almidones nativos de las variedades de ñame D. alata 9506-027, 9403-001, 9605-047, 9506-021 y $9506-025$ para valorarlos como materia prima en aplicaciones industriales alimentarias y no alimentarias, que contribuya a evitar la desaparición del germoplasma de estas variedades, por falta de alternativas de comercialización y agroindustrialización viables que incentiven su cultivo en Colombia.

\section{MÉTODO}

Los rizomas de ñame $D$. alata, rotulados como 9506027, 9403-001, 9605-047, 9506-021 y 9506-025 que pertenecen al banco de germoplasma de la Costa Atlántica Colombiana que comprenden los departamentos de Córdoba, Sucre y Bolívar fueron suministrados por la Universidad de Córdoba, Colombia. La obtención del almidón de ñame se realizó en forma manual y consistió en el lavado, pelado, rallado de los rizomas, extracción con agua, sedimentación y secado artificial. El material rallado se suspendió en una solución de amoniaco $(0,03 \mathrm{M})$ con el objetivo de retirar los mucílagos [8]. El almidón se secó a $40^{\circ} \mathrm{C}$ hasta que se obtuvo una humedad de $10-12 \%$ en base seca.

\section{Análisis proximal}

Se utilizaron métodos basados en las normas AOAC de los Métodos Oficiales de Análisis [9]. La determinación de cenizas según método 945.45 ; humedad por el método 925.10; extracto etéreo método 7.060, 920.39; proteína total según método Kjeldahl 2001.11 y fibra bruta por el método 930.20. El contenido de amilosa se determinó por la norma ISO 6647-1 [10].

\section{Determinación de propiedades físicas y funcionales}

Capacidad de retención de agua (CRA). En un tubo de centrifuga graduado previamente pesado, se depositó aproximadamente un gramo $(1,0 \mathrm{~g})$ de mues- 
tra, se adicionaron $10 \mathrm{~mL}$ de agua destilada y se agitó con suavidad hasta homogenizar. Luego se deja reposar y centrifuga a $3500 \mathrm{rpm}$ durante $15 \mathrm{~min}$. El líquido sobrenadante es decantado y el tubo con el precipitado se drena, durante 10 minutos para luego ser pesado. La ganancia en masa, es la capacidad de absorción de agua de la muestra.

$\mathrm{pH}$ y conductividad eléctrica. Al sobrenadante de suspensiones acuosas al 10\% ( $\mathrm{p} / \mathrm{v}$ ) de almidón, se le determino el $\mathrm{pH}$ y la conductividad eléctrica, usando un pHmetro 691 Metroh y un conductímetro multi-parámetro WTW Multi 340i.

Solubilidad. En la cuantificación de la solubilidad en agua fría y caliente se preparó una muestra de 15 $\mathrm{g}$ de suspensión de almidón al 10\% ( $\mathrm{p} / \mathrm{v})$, se agitó manualmente en un tubo de ensayo, seguido de una centrifugación a $3500 \mathrm{rpm}$ por $15 \mathrm{~min}$ a $30^{\circ} \mathrm{C}, 50^{\circ} \mathrm{C}$ y $70^{\circ} \mathrm{C}$. La masa de líquido sobrenadante se pesó, se trasfirieron $5 \mathrm{~g}$ a una cápsula de porcelana y se procedió al secado en una estufa con recirculación de aire a $105^{\circ} \mathrm{C}$ hasta peso constante, la solubilidad se determinó por la ecuación (Ec.1):

$$
\begin{aligned}
& \text { Masa sólida en la capsula }
\end{aligned}
$$

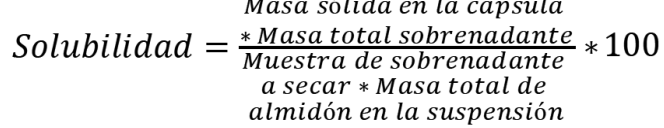

Características reólogicas de suspensiones de almidón . Se prepararon suspensiones de almidón al 8\% $(\mathrm{p} / \mathrm{v})$, el comportamiento reológico fue determinado con un viscosímetro Haake Rotovisco RV20, aplicando deformaciones cada $50 \mathrm{rpm}$ en un rango de $50 \mathrm{rpm}$ hasta $500 \mathrm{rpm}$, en forma ascendente y luego en forma descendente, tomándose lecturas de la tensión tangencial $(t)$ a intervalos de tiempo de un minuto. Las lecturas de esfuerzo cortante se hicieron a $50^{\circ} \mathrm{C}$ y $70^{\circ} \mathrm{C}$.

Comportamiento de la pasta en frio y caliente. Se prepararon suspensiones con $8 \%(\mathrm{p} / \mathrm{v})$ de almidón, se midió de manera continua la viscosidad durante su cocción y enfriamiento en un viscosímetro (Haake Rotovisco RV20). La suspensión de almidón, se calentó en la cápsula del sistema, con un sensor NV agitando a velocidad constante $(350 \mathrm{rpm})$ hasta $50^{\circ} \mathrm{C}$ a una velocidad de calentamiento de aproximadamente $1,5^{\circ} \mathrm{C}$ $\mathrm{min}^{-1}$. Seguidamente, se llevó a $90^{\circ} \mathrm{C}$ por $20 \mathrm{~min}$, se enfríó hasta $50^{\circ} \mathrm{C}$ a una velocidad de $1,5^{\circ} \mathrm{C} \mathrm{min}{ }^{-1}$, per- maneciendo a $50^{\circ} \mathrm{C}$ por 20 min. Información que es utilizada para la construcción de los viscoamilogramas.

Resistencia a la congelación. En la evaluación de la resistencia a la congelación, se prepararon geles al $2 \%(\mathrm{p} / \mathrm{v})$ de almidón, se empacaron en bolsas plásticas, luego se congelaron a $-10^{\circ} \mathrm{C}$ durante dos semanas. En tubos de ensayo se depositaron $5 \mathrm{~g}$ de gel, se centrifugaron a $360 \mathrm{rpm}$ durante 15 min para medir la sinéresis. La sinéresis fue determinada como el porcentaje de la ración de masa separada del líquido en la porción de gel inicial.

\section{Diseño experimental}

Se aplicó un diseño experimental completamente al azar con arreglo unifactorial categórico con cinco niveles correspondientes a las especies de ñame (9506027, 9403-001, 9605-047, 9506-021 y 9506-025) y tres repeticiones para un total de 15 unidades experimentales. Para el análisis de medias, se utilizó el test se Tukey con diferencias significativas a un nivel del $5 \%$, mediante el software Statgraphic Plus versión 5.0.

\section{RESULTADOS}

\section{Análisis proximal}

Los resultados del análisis proximal en base seca (Cuadro 1) indican que existieron diferencias significativas $(P<0,05)$ entre los componentes de los almidones nativos de $D$. alata, que presentan una pureza entre 92 y $96 \%$, resultado próximo al $94,0 \%$ reportado por Hurtado et al; Hurtado y Dufour [11] y al de ñame D. trífida (91,45\%) [12] e inferiores al ñame comercial, blanco y purpura con resultados entre 99,71 a 99,9\% [13].

El valor del contenido de amilosa mostró diferencias significativas $(P<0,05)$, entre todas las variedades estudiadas y su valor es comparado con almidones de ñame $D$. alata cv Diamante $22(22,26 \%)$ y yuca con un valor del $17,93 \%$ [14] y a los reportados para Dioscorea sp por [15, 16]. El contenido de amilosa en la variedad $D$. alata es alto comparado con las especies de ñame $D$. trífida y $D$. esculenta que le otorgan al almidón características de geles y lo hace adecuado para modificaciones enzimáticas que permitan obtener geles termorreversibles y resistentes $[17,18]$. Por lo general almidones con bajo contenido de ami- 
losa son más cristalinos y digestibles, mientras que almidones con alto contenido a amilosa son menos susceptibles a la degradación enzimática. Además, la digestibilidad depende del tipo de cristalinidad del almidón, que para el caso del ñame es tipo B, que los potencializa hacia el desarrollo de almidones resistentes [19, 20, 21]. Se observa que los valores nutricionales (proteína, extracto etéreo, fibra y cenizas) son bastante bajos, debido a que gran parte se pierde durante el proceso de extracción.

\section{Propiedades funcionales}

En el (Cuadro 2), se observa que la CRA varían significativamente $(P<0,05)$ y es menor a la reportada por Espitia y Salcedo [6] en almidones de D. Bulbífera, D. Trífida y D. Esculenta y son superiores a almidones oxidados de yuca [22]. Esta propiedad es importante para mejorar los rendimientos en productos elaborados por la industria alimentaria (embutidos cárnicos), en los procesos de calefacción óhmica para cocinar alimentos ricos en almidón [23].

Los almidones estudiados presentaron en promedio un rango de $\mathrm{pH}$ neutro, que favorece su aplicación en mezclas con otros tipos de alimentos. La variedad 9506-
021 mostró el mayor valor de conductividad eléctrica, mientras que la variedad 9403-001 registró el menor valor. Estos valores son inferiores al valor registrado por el almidón oxidado de yuca de 190,75 $\mu \mathrm{s} / \mathrm{cm}[6]$. La estabilidad y el pH de las emulsiones cárnicas son dependientes del tipo de extensor y el porcentaje de inclusión [24]. La introducción de almidón puede sustituir productos ricos en proteínas utilizados como extensores.

Las solubilidades de los almidones de ñame a 30 , 50 y $70^{\circ} \mathrm{C}$ presentaron diferencias estadísticamente significativas $(P<0,05)$. En todas las variedades de ñame estudiadas la solubilidad aumento con la temperatura, se debe a que los gránulos hinchados del almidón permiten una mayor la exudación de amilosa [22], siendo inferiores a las variedades de D. alata, D. rotundata y yuca [6,25]; almidón céreo de ñame, en almidón de maíz acetilado [13], ñame D. trífida [12], cebada, almidón oxidado de cebada [4] amaranto, en amaranto modificado con tratamiento acido, maíz céreo fosfatado y succinado [26]. Es importante la solubilidad del almidón en productos elaborados que requieran de tratamientos térmicos menores de $80^{\circ} \mathrm{C}$ (néctares pasteurizados a $72^{\circ} \mathrm{C}$ ). Los almidones nativos de ñame ( $D$. alata) por tener bajas solubilidades no se podrían aplicar en este tipo de productos. La

Cuadro 1. Análisis composicional de almidones nativos de ñame.

\begin{tabular}{|l|c|c|c|c|c|}
\hline \multicolumn{1}{|c|}{ Componentes (\%) } & $9506-027$ & $9403-001$ & $9605-047$ & $9506-021$ & $9506-025$ \\
\hline M. Seca & $88,77 \pm 0,01 a$ & $87,65 \pm 0,01 b$ & $89,24 \pm 0,00 c$ & $87,99 \pm 0,008 b$ & $89,03 \pm 0,008 c$ \\
\hline Almidón & $95,00 \pm 0,81 a$ & $94,00 \pm 0,81 b$ & $96,00 \pm 0,85 c$ & $96,00 \pm 0,85 c$ & $92,00 \pm 0,81 d$ \\
\hline Amilosa & $24,08 \pm 0,01 a$ & $25,60 \pm 0,00 b$ & $23,20 \pm 0,01 c$ & $24,86 \pm 0,01 d$ & $25,44 \pm 0,01 e$ \\
\hline Cenizas & $0,20 \pm 0,04 a$ & $0,10 \pm 0,04 b$ & $0,09 \pm 0,00 c$ & $0,26 \pm 0,00 d$ & $0,10 \pm 0,016 b$ \\
\hline Proteína & $0,50 \pm 0,08^{a}$ & $0,40 \pm 0,01 b$ & $0,50 \pm 0,01^{a}$ & $0,60 \pm 0,10 c$ & $0,60 \pm 0,04 c$ \\
\hline Extracto Etéreo & $0,60 \pm 0,08 a$ & $0,70 \pm 0,05 b$ & $0,50 \pm 0,10 c$ & $0,60 \pm 0,08^{a}$ & $0,90 \pm 0,03 d$ \\
\hline Fibra & $0,225 \pm 0,09^{a}$ & $0,30 \pm 0,08 b$ & $0,30 \pm 0,12 b$ & $0,40 \pm 0,08 c$ & $0,50 \pm 0,07 d$ \\
\hline
\end{tabular}

Cuadro 2. Propiedades físicas y funcionales de almidones nativos de ñame.

\begin{tabular}{|l|c|c|c|c|c|}
\hline \multicolumn{1}{|c|}{ Propiedades } & $9506-027$ & $9403-001$ & $9605-047$ & $9506-021$ & $9506-025$ \\
\hline $\mathrm{CRA} \%\left(30^{\circ} \mathrm{C}\right)$ & $87,81 \pm 0,61 \mathrm{a}$ & $99,49 \pm 0,24 \mathrm{~b}$ & $106,23 \pm 0,56 \mathrm{c}$ & $117,71 \pm 0,34 \mathrm{~d}$ & $98,64 \pm 0,40 \mathrm{e}$ \\
\hline $\mathrm{pH}$ & $7,33 \pm 0,02 \mathrm{a}$ & $7,25 \pm 0,03 \mathrm{a}$ & $6,85 \pm 0,04 \mathrm{~b}$ & $6,76 \pm 0,03 \mathrm{~b}$ & $6,85 \pm 0,02 \mathrm{~b}$ \\
\hline Conductividad eléctrica $\mu \mathrm{s} / \mathrm{cm}$ & $23,30 \pm 3,10 \mathrm{a}$ & $16,00 \pm 0,88 \mathrm{~b}$ & $75,82 \pm 0,29 \mathrm{c}$ & $79,10 \pm 0,26 \mathrm{~d}$ & $48,58 \pm 2,20 \mathrm{e}$ \\
\hline$\%$ Solubilidad $\left(30^{\circ} \mathrm{C}\right)$ & $0,43 \pm 0,01 \mathrm{a}$ & $0,72 \pm 0,01 \mathrm{~b}$ & $1,58 \pm 0,11 \mathrm{c}$ & $1,30 \pm 0,03 \mathrm{~d}$ & $0,94 \pm 0,00 \mathrm{e}$ \\
\hline$\%$ Solubilidad $\left(50^{\circ} \mathrm{C}\right)$ & $0,59 \pm 0,02 \mathrm{a}$ & $0,85 \pm 0,01 \mathrm{~b}$ & $1,92 \pm 0,06 \mathrm{c}$ & $1,61 \pm 0,05 \mathrm{~d}$ & $1,20 \pm 0,05 \mathrm{e}$ \\
\hline$\%$ Solubilidad $\left(70^{\circ} \mathrm{C}\right)$ & $1,12 \pm 0,12 \mathrm{a}$ & $1,10 \pm 0,10 \mathrm{a}$ & $2,12 \pm 0,04 \mathrm{~b}$ & $2,15 \pm 0,03 \mathrm{~b}$ & $1,44 \pm 0,00 \mathrm{c}$ \\
\hline Viscosidad aparente $\mathrm{cp}\left(50^{\circ} \mathrm{C}\right) \times 10-3$ & $1,15 \pm 0,04 \mathrm{a}$ & $2,00 \pm 0,21 \mathrm{~b}$ & $3,5 \pm 0,08 \mathrm{c}$ & $3,50 \pm 0,04 \mathrm{c}$ & $2,50 \pm 0,08 \mathrm{~d}$ \\
\hline viscosidad aparente $\mathrm{cp}\left(70^{\circ} \mathrm{C}\right) \times 10-3$ & $2,00 \pm 0,16 \mathrm{a}$ & $2,50 \pm 0,01 \mathrm{~b}$ & $3,5 \pm 0,07 \mathrm{c}$ & $3,50 \pm 0,08 \mathrm{c}$ & $3,50 \pm 0,24 \mathrm{c}$ \\
\hline$\%$ Sinéresis & $58,30 \pm 0,24 \mathrm{a}$ & $60,40 \pm 0,29 \mathrm{~b}$ & $61,6 \pm 0,18 \mathrm{~b}$ & $63,20 \pm 0,18 \mathrm{c}$ & $59,10 \pm 0,08 \mathrm{a}$ \\
\hline
\end{tabular}


solubilidad baja solubilidad se relaciona con el contenido de amilosa [27] y al tamaño del granulo, en este caso los resultados mostraron contenido de amilosa que es medianamente alto para almidones nativos.

La evaluación de la estabilidad a la refrigeración y congelación consiste en verificar en los geles la sinéresis como consecuencia de la reorganización de las moléculas del almidón. La variedad 9506-021 presentó el mayor valor de sinéresis mientras que la variedad 9506-027 registró el menor valor. Estos almidones tienen menores valores de sinéresis con respecto a las especies $D$. alata y $D$. rotundata [16], similar a almidones nativos $D$. trífida, D. esculenta, $D$. bulbifera [6] y menos estables con respecto al almidón de papa criolla reportado por Polanco et al. [28]. Los almidones estudiados en este trabajo, presentaron una mediana resistencia a la congelación, muestran geles muy rígidos originados por su fuerte retrogradación, ligada a los elevados contenidos de amilosa que poseen. De acuerdo a estos resultados, los almidones de ñame pueden ser utilizados en aquellos alimentos que requieren ser almacenados en refrigeración y necesitan cierta exudación de humedad para proporcionar una apariencia fresca, como los flanes o salsas.

La Figura 1, muestra los viscoamilogramas de los almidones nativo de ñame, cuando se sometieron las diferentes suspensiones de almidón al calentamiento y enfriamiento controlado. El comportamiento de las suspensiones de los almidones nativos de las diferentes variedades de $D$. alata durante su calentamiento y posterior enfriamiento, muestra que el almidón de la variedad 9506-027 posee el mayor poder espesante de los cinco almidones al presentar el mayor pico de viscosidad a $93^{\circ} \mathrm{C}$ seguido por la variedad 9403-001, los restantes almidones no presentaron picos de viscosidad bien definidos. Los almidones $D$. alata refe-

Figura 1. Viscoamilograma de los almidones nativos de ñame (D. alata).

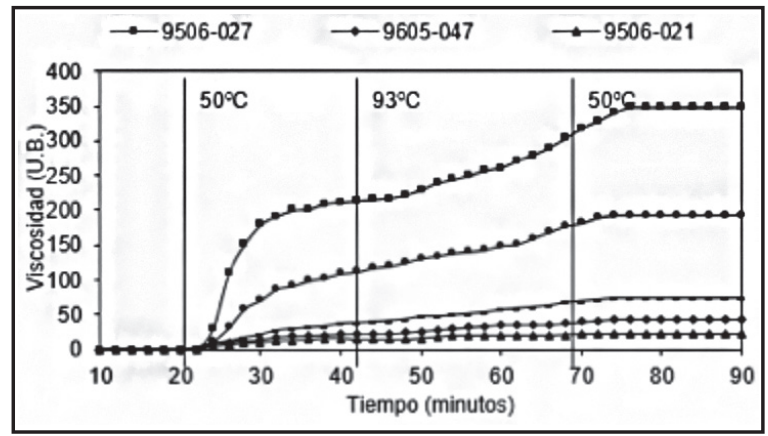

renciados en esta investigación (Cuadro 3) presentan un valor de viscosidad máxima menor que almidones nativos y acetilados de yuca y ñame [14], harina de ñame [29] variabilidad asociada a la estructura de los almidones [3]. Los almidones de D. alata presentaron una viscosidad estable cuando son enfriados, resultado que debe tenerse en cuenta al momento de pensar en incorporar el almidón a algún producto que necesite enfriamiento por largos tiempos, debido a la posibilidad de separación de fases por sinéresis.

Las temperaturas de gelatinización variaron entre 83 y $84,2^{\circ} \mathrm{C}$, fueron superiores a temperaturas de gelatinización presentadas por otros almidones como el de la oca amarilla $\left(48,1^{\circ} \mathrm{C}\right)$, maíz céreo $\left(72,2^{\circ} \mathrm{C}\right)$, yuca $\left(65^{\circ} \mathrm{C}\right)$, Sagú $\left(74,5^{\circ} \mathrm{C}\right)$ y papa $\left(58\right.$ a $\left.68^{\circ} \mathrm{C}\right)[15,18$, $22,29]$, arroz $\left(68-74-78^{\circ} \mathrm{C}\right)$, maíz $\left(62-67-72^{\circ} \mathrm{C}\right)$, yuca $\left(59-64-69^{\circ} \mathrm{C}\right)$, sorgo $\left(68-74-78^{\circ} \mathrm{C}\right)$ y a las de ñame genotipo amazónico blanco $\left(76,8-77^{\circ} \mathrm{C}\right)$, purpura claro $\left(75,5-76,9^{\circ} \mathrm{C}\right)$ y purpura oscuro $\left(77-80^{\circ} \mathrm{C}\right)[13]$.

Los almidones de D. alata al mostrar una gran estabilidad (breakdown) a los esfuerzos de corte durante el calentamiento, definen su aplicación en productos que tengan que ser sometidos a procesos de esterilización, escaldado y productos de confitería. Además, presentan valores positivos del setback, indicando un aumento de la viscosidad que se relaciona con el restablecimiento de los enlaces hidrógeno de amilosa y amilopectina formando una consistencia tipo gel. Asimismo, el incremento de la viscosidad durante el enfriamiento, indica una tendencia de los componentes a la retrogradación, que puede ser importante en la aplicación de almidones resistentes a la acción de enzimas amilolíticas [31]. En ese orden, la variedad 9506-027 tiene mayor tendencia a retrogradar, por lo que su utilización en productos alimenticios que debe ser almacenado en frio es limitada, para incorporar los almidones de ñame en este tipo de productos, se debe modificar el almidón de tal forma que conserve su estabilidad y disminuya la retrogradación.

\section{Comportamiento reológico}

Los reogramas del esfuerzo de cizalla $(\sigma)$ versus el gradiente de velocidad $(\gamma)$ de los almidones $D$. alata, se muestran en la Figura 2. Estos, exhibieron un comportamiento no tixotrópico, su viscosidad aparente decrece al aumentar la velocidad de corte, caracterizándose como fluidos no newtonianos tipo pseudoplásticos. Se han reportado comportamientos pseudoplásticos en estudio de índice de flujo para almidón 
Cuadro 3. Resultados de viscoamilogramas de almidones de D. alata

\begin{tabular}{|l|l|l|l|l|l|l|}
\hline \multicolumn{1}{|c|}{ Variedad } & \multicolumn{1}{|c|}{ T gel $^{\circ} \mathbf{C}$} & \multicolumn{1}{|c|}{ V93 (U.B) } & \multicolumn{1}{c|}{ V93/20 (U.B) } & \multicolumn{1}{c|}{ V50 (U.B) } & \multicolumn{1}{c|}{ Setback } & \multicolumn{1}{c|}{ Breakdown } \\
\hline $9506-027$ & 83,75 & 170 & 226 & 348 & 178 & -56 \\
\hline $9403-001$ & 83 & 66 & 129 & 194 & 128 & -63 \\
\hline $9605-047$ & 83,75 & 13 & 25 & 44 & 31 & -12 \\
\hline $9506-021$ & 84,2 & 9 & 15 & 22 & 13 & -6 \\
\hline $9506-025$ & 83 & 20 & 46 & 55 & 35 & -26 \\
\hline
\end{tabular}

T gel: temperatura gelatinización; V93: viscosidad a $93^{\circ} \mathrm{C}$; V93/20: viscosidad a $93^{\circ} \mathrm{C}$ por 20 min; V50: viscosidad a $50^{\circ} \mathrm{C}$; Setback: asentamiento; Breakdown: estabilidad

Figura 2. Comportamiento de los almidones de ñame (D. alata) a esfuerzos de corte.

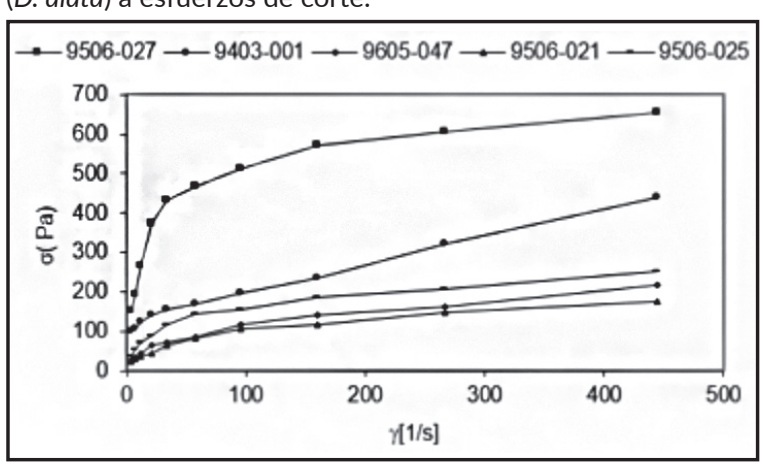

de plátano (Musa ABB) [31]; No newtoniano para almidón de semillas de huauzontle [32] y HerschelBulkley en almidón de yuca reticulado-acetilado [33].

El modelo reológico usado para describir el comportamiento de los almidones de ñame $D$. alata es el modelo de Herschel-Bulckley (Ec. 2).

$$
\sigma=\sigma_{-} o+K \gamma^{\wedge} n
$$

Todos los almidones presentaron un índice de comportamiento de flujo ( $\mathrm{n}$ ) menor a 1 . El índice de consistencia (K) fue mayor en la variedad 9506-027 (Cuadro 4).

Un aumento en la concentración causa un incremento en la pseudoplasticidad mostrando una disminución en el índice de flujo ( $\eta$ y por consiguiente el coeficiente de consistencia $(K)$ se incrementa con la concentración [34], caso que se presenta en las variedades de $D$. alata donde hay mayor pseudoplasticidad en orden de 9506-027>9403-001>9506025>9605-047>9506-021. Este comportamiento se denomina reofluidizante y ocurre cuando el esfuerzo desorganiza el arreglo de las moléculas de la matriz.
Cuadro 4. Parámetros modelo de Herschel-Bulckley.

\begin{tabular}{|l|l|l|l|l|}
\hline \multicolumn{1}{|c|}{ Variedad } & \multicolumn{1}{c|}{$\boldsymbol{\delta}_{\mathrm{o}}(\mathrm{Pa})$} & $\boldsymbol{K}\left(\mathrm{Ns} / \mathbf{m}^{2}\right)$ & \multicolumn{1}{c|}{$\mathbf{N}$} & \multicolumn{1}{c|}{$\mathbf{R}^{2}$} \\
\hline $9506-027$ & 150,4 & 119,03 & 0,3081 & 0,92 \\
\hline $9403-001$ & 97,76 & 56,405 & 0,3014 & 0,95 \\
\hline $9605-047$ & 26,32 & 14,665 & 0,4434 & 0,99 \\
\hline $9506-021$ & 22,56 & 12,314 & 0,4487 & 0,99 \\
\hline $9506-025$ & 37,60 & 25,408 & 0,3919 & 0,97 \\
\hline
\end{tabular}

\section{CONCLUSIONES}

De las propiedades funcionales de las variedades de D. alata, las 9506-021 y 9605-047 presentaron los valores superiores al $100 \%$ de capacidad de retención de agua, posibilitando su uso en la industria alimentaria para el aumento de rendimientos en productos cárnicos, las variedades 9506-027 y 9403001 presentaron las viscosidades más altas durante el proceso de calentamiento y enfriamiento, por lo que serían útiles como espesantes para productos alimentarios ( cremas ligeras, sopas y coladas), y en general todas registraron viscosidades crecientes. Todas las variedades presentaron valores bajos de conductividad eléctrica menores de $80 \mu \mathrm{s} / \mathrm{cm}$. Las solubilidades son inferiores del $3 \%$, limitando el uso estos almidones en productos alimentarios procesados a bajos tratamientos térmicos. Las altas temperaturas de gelatinización presentados por los almidones de $D$. alata los hace importantes en procesos donde se necesiten productos con tratamientos térmicos. Los almidones presentaron sinéresis al ser sometidos a temperaturas a $-10^{\circ} \mathrm{C}$, por lo que no son aptos para la elaboración de productos congelados. Se hace necesario implementar modificaciones químicas o físicas las cinco variedades de almidón de $D$. alata, para lograr extender la gama de aplicaciones industriales en las que podrían ser utilizados. Las cinco variedades de $D$. alata se comportan como fluidos no newtonianos, independientes del tiempo y com- 
portamiento pseudoplástico, ajustándose al modelo de Herschel-Bulckley.

\section{AGRADECIMIENTOS}

Al Programa de Investigación Participativa para la Producción y Transformación Sostenible del Ñame (Dioscorea s.p.) en la Costa Atlántica, Colciencias, Universidad de Córdoba, Universidad de Sucre, Corpoica, Corporación PBA

\section{REFERENCIAS}

[1] AGRONET. Estadísticas agroforestales Consolidado Nacional. 2015 [online].Available: http://www. agronet.gov.co. Accessed: 20-Jul-2017

[2] VARGAS-AGUILAR, P. y HERNÁNDEZ-VILLALOBOS, D. Harinas y almidones de yuca, ñame, camote y ñampí: propiedades funcionales y posibles aplicaciones en la industria alimentaria. Revista Tecnología en Marcha, 26(1), 2013, p. 37-45.

[3] REINA ARANZA, Y.C. El cultivo del Ñame en el caribe colombiano, Documento de trabajo sobre economia regional. Cartagena (Colombia): Banco de la Republica (CEER), 2012.

[4] EL HALAL, S.L.M. et al., Structure, morphology and functionality of acetylated and oxidised barley starches. Food Chemistry, 168, 2015, p. 247-256.

[5] DUPUIS, J.H., LIU, Q. and YADA, R. Y. Methodologies for increasing the resistant starch content of food starches: A review. Compr. Food Science and Food Safety, 39(1), 2014, p. 1219-1234.

[6] ESPITIA, J., SALCEDO, J. y GARCIA, C. Propiedades funcionales de almidones de ñame (Dioscorea bulbifera, Dioscorea trifida y Dioscorea esculenta). Revista Técnica de la Faculatd de Ingeniería de la Universidad de Zulia, 10, 2016, p. 31- 36019.

[7] SALAZAR, E. y MARCANO, M. La Harina de Ñame (Dioscorea Alata), Un Ingrediente Potencial en la Elaboración de Productos de Productos de Panadería. SABER, 23(2), 2012, p 134-140[

8] MOORTHY, S.N. Extraction of starches from tuber crops using ammonia. Carbohydrate Polymer, 16(4), 1991, p. 391-398.

[9] ASSOCIATION OF OFFICIAL ANALYTICAL CHEMISTS (AOAC). Official Method of Analysis, 20th ed, 13th ed. Washington D.C (USA): AOAC International, 2016.
[10] INTERNATIONAL STANDARIZATION ORGANIZATION (ISO). Norma 6647-1. 2 ed. Norma de la organización internacional para la estandarización sobre determinación de amilosa. Génova (Suiza): 2011.

[11] DUFOUR, D. y HURTADO BERMUDEZ, J. Analisis comparativo de las propiedades funcionales de diversos almidones de raices y tuberculos autochtonos de Colombia. Potencialidades de uso. Lima (Perú): Seminario tecnico sobre raices y tuberculos autoctonos, Programa Nacional de Maquinaria y Postcosecha; Programa Regional de Sistemas de Produccion, CORPOICA, 1997, p. 1-21.

[12] ACUÑA-PINTO, H.M. Extracción, caracterización y aplicación de almidón de ñame variedad blanco (Dioscorea trifida) originario de la región amazónica colombiana para la elaboración de productos horneados [Tesis Especialiste en Ciencia y Tecnología de Alimentos]. Bogotá (Colombia): Universidad Nacional de Colombia, Facultad de Ciencias, 2012, $80 \mathrm{p}$.

[13] PÉREZ, E. et al. Physicochemical, functional, and macromolecular properties of waxy yam starches discovered from 'Mapuey'(Dioscorea trifida) genotypes in the Venezuelan Amazon. Journal of Agricultural and Food Chemistry, 59(1), 2010, p. 263-273.

[14] FIGUEROA, J., SALCEDO, J. and RODRIGUEZ, M. Acetylated cassava and yam starches for food use. Agronomía Colombiana (supl), 34(1), 2016 , p. 785-788.

[15] FEN, Z. Isolation, composition, structure, properties, modifications, and uses of yam starches. Coomprehensive review in food sciencie and food safety, 14, 2015, p. 257-386.

[16] ODEKUA, O.A. and PICKER-FREYER, K. Analysis of the material and tablet formation properties of four Dioscorea starches. Starch-Stärke, 59(9), 2007, p. 430-444.

[17] KAUR, B., ARIFFIN, F., BHAT, R. and KARIM, A. Progress in starch modification in the last decade. Food Hydrocolloids, 26(2), 2012, p. 398-404.

[18] TRINH, K.S., CHOI, S.J. and MOON, T. Structure and digestibility of debranched and hydrothermally treated water yam starch. Starch-Stärke, 65(7-8), 2013, p. 679-685.

[19] APRIEANITA, A., VASILJEVIC, T., BANNIKOVA, A. and KASAPIS, S. Physicochemical properties of flours and starches derived from traditional Indonesia tuber and root. Journal of Food Science and Technology, 51(12), 2014, p. 3669-3679. 
[20] SALCEDO, J., RODRIGUEZ, M. y FIGUEROA, J. Efecto de la acetilación en las propiedades estructurales y funcionales de almidones de yuca y ñame. Revista Mexicana de ingeniería química, 15(3), 2016, p. 787-796.

[21] WANG, S. et al. Starch retrogradation: A comprehensive review. Comprehensive reviews in food safety, 14, 2015, p. 568-585.

[22] ASCENCIO, M., ANDRADE, R. y SALCEDO, J. Caracterización funcional de almidón oxidado de yuca (Manihot esculenta). Agronomía Colombiana (supl), 34(1), 2016, p. 757-759.

[23] WEERACHET, J. et al. Ohmic heating for cooking rice: Electrical conductivity measurements, textural quality determination and energy analysis. Innovative Food Science and Emerging Technologies, 42, 2017, p 16-24.

[24] ALVARRACIN, W., DELGADO, N. y VILLEGAS, C. Efecto de la inclusión de diferentes extensores sobre parámetros fisicoquímicos de emulsiones cárnicas. Agronomía Colombiana (supl), 34(1), 2016, p. 215-218.

[25] GUIZAR, M.A., MIRANDA, J.L, SOTO, M. y RUIZ, I. Parcial caracterización de nuevos almidones obtenidos del tubérculo de camote del cerro (Dioscorea spp). Revista Iberoamericana de Tecnología Postcosecha, 9(1), 2008, p. 81-88.

[26] GARCÍA-RODRÍGUEZ, A.P. Obtención y caracterización de almidones modificados y su aplicación como agentes encapsulantes del bioinsecticida Bacillus thuringiensis [Tesis Ingeniero Químico de Alimentos]. Querétaro (Mexico): Universidad de Querétaro, Facultad de Química, 2011, 141 p.

[27] HUNG, P., VIEN, N. and LAN-PHI, N. Resistant starch improvement of rice starches under a combination of acid and heat-moisture treatments. Food Chemistry, 191(15), 2016, p 67-73.

[28] POLANCO, L.Z. et al. Extracción y caracterización de almidón nativo de clones promisorios de papa criolla (Solanum tuberosum, Grupo Phureja). Revista Latinoamericana de la Papa, 18(1), 2014, p. 1-24.

[29] PATERNINA, A., SALCEDO, J. y ROMERO, P. Efecto de la harina de ñame ( $D$. rotundata) sobre las propiedades texturales de salchichas. Agronomía Colombiana (supl), 34(1), 2016, p. 379- 381.

[30] WANG, S. et al. Starch retrodegradattion: A comprehesive review. Comprehesive review in food sciences and food safety, 14, 2015, p. 568-585.

[31] BELLO, J. et al. Extracción y caracterización reologica del almidon y pectina frutos de platano " pera" (Musa ABB). Revista Mexicana de Ciencias Agrícolas, 8, 2014, p. 1501- 1507.

[32] ASSAD, M. et al. Caracterización reológica, térmica, funcional y fisicoquímica del almidón de semillas de huauzontle (Chenopodium berlandieri spp. nuttalliae). Agrociencia, 48(8), 2014, p. 789-803

[33] CUI, B. et al. Effect of cross-linked acetylated starch content on the structure and stability of set yoghurt. Food Hydrocolloids, 35, 2014, p. 576-582.

[34] CEVOLI, C., BALESTRA, F., RAGNI, L. and FAB$B R I, A$. Rheological characterisation of selected food hydrocolloids by traditional and simplified techniques. Food Hydrocolloids, 33(1), 2013, p. 142-150. 